Pacific Journal of Mathematics

ZERO SQUARE RING

richard Peter Stan dey 


\section{ZERO SQUARE RINGS}

\section{Richard P. Stanley}

A ring $R$ for which $x^{2}=0$ for all $x \in R$ is called a zerosquare ring. Zero-square rings are easily seen to be locally nilpotent. This leads to two problems: (1) constructing finitely generated zero-square rings with large index of nilpotence, and (2) investigating the structure of finitely generated zerosquare rings with given index of nilpotence. For the first problem we construct a class of zero-square rings, called free zero-square rings, whose index of nilpotence can be arbitrarily large. We show that every zero-square ring whose generators have (additive) orders dividing the orders of the generators of some free zero-square ring is a homomorphic image of the free ring. For the second problem, we assume $R^{n} \neq 0$ and obtain conditions on the additive group $R_{+}$of $R$ (and thus also on the order of $R$ ). When $n=2$, we completely characterize $R_{+}$. When $n>3$ we obtain the smallest possible number of generators of $R_{+}$, and the smallest number of generators of order 2 in a minimal set of generators. We also determine the possible orders of $R$.

Trivially every null ring (that is, $R^{2}=0$ ) is a zero-square ring. From every nonnull commutative ring $S$ we can make $S \times S \times S$ into a nonnull zero square ring $R$ by defining addition componentwise and multiplication by

$$
\left(x_{1}, y_{1}, z_{1}\right) \times\left(x_{2}, y_{2}, z_{2}\right)=\left(0,0, x_{1} y_{2}-x_{2} y_{1}\right) .
$$

In this example we always have $R^{3}=0$. If $S$ is a field, then $R$ is an algebra over $S$. Zero-square algebras over a field have been investigated in [1].

2. Preliminaries. Every zero-square ring is anti-commutative, for $0=(x+y)^{2}=x^{2}+x y+y x+y^{2}=x y+y x$. From anti-commutativity we get $2 R^{3}=0$, for $y z x=y(-x z)=-(y x) z=x y z$ and $(y z) x=-x(y z)$, so $2 x y z=0$ for all $x, y, z \in R$. It follows that a zero-square ring $R$ is commutative if and only if $2 R^{2}=0$.

If $R$ is a zero-square ring with $n$ generators, then any product of $n+1$ generators must contain two factors the same. By applying anti-commutativity we get a square factor in the product; hence $R^{n+1}=0$. In particular, every zero-square ring is locally nilpotent.

If $G$ is a finitely generated abelian group, then by the fundamental theorem on abelian groups we have

$$
\begin{aligned}
G & =C_{a_{1}} \oplus \cdots \oplus C_{a_{n}}, a_{i} \mid a_{i+1} \text { for } 1 \leqq i \leqq k-1, \\
a_{k+1} & =\cdots=a_{n}=\infty,
\end{aligned}
$$


where $C_{a_{i}}$ is a cyclic group of order $a_{i}$. If $X=\left\{x_{1}, \cdots, x_{n}\right\}$ generates $G$ and if there is some decomposition (1) for which $x_{i}$ generates $C_{a_{i}}$, $1 \leqq i \leqq n$, then we call $X$ a standard set of (group) generators for $G$. Now let $R$ be any finitely generated ring with a minimal set of ring generators $X^{\prime}=\left\{x_{1}, \cdots, x_{n}\right\}$. Let $\left\langle X^{\prime}\right\rangle$ denote the additive group generated by $X^{\prime}$ (whose elements are considered now as group, not ring, generators), and let $X$ be a standard set of generators for $\left\langle X^{\prime}\right\rangle$. Then $X$ generates $R$ as a ring since it generates $\left\langle X^{\prime}\right\rangle$ as a group. Such a set $X$ will be called a standard set of ring generators for $R$, and it follows that every finitely generated ring has a standard set of ring generators.

3. Free zero-square rings. For every positive integer $n$ and every $n$-tuple $\left(a_{1}, \cdots, a_{n}\right)$, where $a_{i} \mid a_{i+1}$ for $i=1, \cdots, k-1$, and $a_{k+1}=\cdots=a_{n}=\infty$, we define the free zero-square $\operatorname{ring} R_{F}\left(a_{1}, \cdots, a_{n}\right)$ and derive its basic properties. Free zero-square ring are constructed from combinations of indeterminates called special monomials.

DEFINITION 3.1. Let $a_{1}, \cdots, a_{n}$ be integers $\geqq 2$ or $\infty$, such that for some $k \leqq n, a_{i} \mid a_{i+1}$ for $i=1, \cdots, k-1$, while $a_{k+1}=\cdots=a_{n}=\infty$; and let $x_{1}, \cdots, x_{n}$ be indeterminates. We say that $x_{i_{1}} x_{i_{2}} \cdots x_{i_{q}}$ is a special monomial if $1 \leqq i_{1}<i_{2}<\cdots<i_{q} \leqq n$, and if $a_{i_{1}}$ is even or $\infty$ whenever $q>2$.

Thus the special monomials consist of

$$
\begin{aligned}
x_{i}, & 1 \leqq i \leqq n \\
x_{i} x_{j}, & 1 \leqq i<j \leqq n \\
x_{i_{1}} x_{i_{2}} \cdots x_{i_{q}}, & q \geqq 3 \text { and } a_{i_{1}} \text { even or } \infty .
\end{aligned}
$$

Now let $y_{1}, y_{2}, \cdots, y_{r}$ denote the $r$ distinct special monomials (in some order) corresponding to $a_{1}, a_{2}, \cdots, a_{n}$. If $y_{j}=x_{i_{1}} x_{i_{2}} \cdots x_{i_{q}}$ is a special monomial, we define

$$
b_{j}=b\left(y_{j}\right)= \begin{cases}a_{i_{1}}, & \text { if } q=1 \text { or } 2 \\ 2, & \text { if } q \geqq 3\end{cases}
$$

Let $R_{F}\left(a_{1}, \cdots, a_{n}\right)$ denote the set of formal sums

$$
\begin{gathered}
R_{F}\left(a_{1}, \cdots, a_{n}\right)=\left\{\sum_{i=1}^{r} c_{i} y_{i} \mid 0 \leqq c_{j}<b_{j} \text { if } b_{j} \neq \infty,\right. \\
\left.-\infty<c_{j}<\infty \text { if } b_{j}=\infty\right\} .
\end{gathered}
$$

We define addition and multiplication on $R_{F}$ as follows: 
Addition. Define

$$
\sum_{i=1}^{r} c_{i} y_{i}+\sum_{i=1}^{r} d_{i} y_{i}=\sum_{i=1}^{r} e_{i} y_{i},
$$

where $e_{i} \equiv c_{i}+d_{i}\left(\bmod b_{i}\right), 0 \leqq e_{i}<b_{i}$ if $b_{i} \neq \infty, e_{i}=c_{i}+d_{i}$ if $b_{i}=\infty$. We are adding the $i^{\text {th }}$ components $\bmod b_{i}$.

Multiplication. We first define multiplication of special monomials. If $y_{i}$ and $y_{j}$ have a factor $x_{s}$ in common, define $y_{j} y_{i}=y_{i} y_{j}=0$. In particular, $x_{s}^{2}=0$. If $y_{i}=x_{s}, y_{j}=x_{t}$ with $s<t$, define $\left(a y_{i}\right)\left(b y_{j}\right)=$ $\overline{a b} x_{s} x_{t}$; where if $b_{i} \neq \infty$, then $\overline{a b}$ is defined by $\overline{a b} \equiv a b\left(\bmod b_{i}\right), 0 \leqq \overline{a b}<b_{i}$, while if $b_{i}=\infty$, then $\overline{a b}=a b$. If we think of $a$ and $b$ as representatives of the congruence classes $\bmod b_{i}$ and $b_{j}$, then since $b_{i} \mid b_{j}$ the product $a b$ always represents the same element $\bmod b_{i}$ regardless of the choice of $a$ and $b$. Similarly define $\left(b y_{j}\right)\left(a y_{i}\right)=\overline{-a b}\left(x_{s} x_{t}\right)$. If $y_{i}$ and $y_{j}$ do not have a factor $x_{s}$ in common, and if at least one of $y_{i}, y_{j}$ contains at least two distinct factors $x_{s}$ and $x_{t}$, then define $\left(a y_{i}\right)\left(b y_{j}\right)=c y_{l}$, where $y_{l}$ is obtained by rearranging the factors $x_{h}$ of $y_{i}$ and $y_{j}$ in ascending subscript order and defining

$$
c=\left\{\begin{array}{l}
0, \text { if } a_{q} \text { is odd } \\
0, \text { if } a_{q} \text { is even or } \infty \text { and } a b \text { is even } \\
1, \text { if } a_{q} \text { is even or } \infty \text { and } a b \text { is odd },
\end{array}\right.
$$

where $a_{q}$ is the order of the indeterminate $x_{q}$ with least subscript appearing in $y_{l}$.

We now define in general

$$
\left(\sum_{i} c_{i} y_{i}\right)\left(\sum_{j} d_{j} y_{j}\right)=\sum_{i, j}\left(c_{i} y_{i}\right)\left(d_{j} y_{j}\right)
$$

where this sum is to be rearranged according to the previously defined rules of special monomial multiplication and of addition.

We call this set $R_{F}\left(a_{1}, \cdots, a_{n}\right)$, together with the operations of addition and multiplication just defined, the free zero square ring $R_{F}\left(a_{1}, \cdots, a_{n}\right)$.

Theorem 3.2. $R_{F}\left(a_{1}, \cdots, a_{n}\right)$ is a zero-square ring.

Proof. All the desired properties follow from the definitions except associativity of multiplication and the zero-square property.

It follows from the definition of multiplication that we need only to verify associativity for monomials $c_{h} y_{h}$, where $c_{h}$ is a constant between 0 and $b_{h}-1$ for $b_{h} \neq \infty$, while $-\infty<c_{h}<\infty$ for $b_{h}=\infty$, 
and $y_{h}$ is a special monomial. But if either of $y_{h}, y_{i}, y_{j}$ contain an indeterminate $x_{s}$ of odd order, then $\left(c_{h} y_{h}\right)\left(c_{i} y_{i}\right)\left(c_{j} y_{j}\right)=0$ upon any association, while if all orders are even or $\infty$, then

$$
\left(c_{h} y_{h}\right)\left(c_{i} y_{i}\right)\left(c_{j} y_{j}\right)=\left\{\begin{array}{c}
0, \text { if two of } y_{h}, y_{i}, y_{j} \text { contain } \\
\text { a common factor } x_{e} \\
0, \text { if any of } c_{h}, c_{i}, c_{j} \text { is even } \\
y_{h} y_{i} y_{j}, \text { otherwise }
\end{array}\right.
$$

upon any association.

It remains only to show $\left(\sum c_{i} y_{i}\right)^{2}=0$. Now

$$
\left(\sum c_{i} y_{i}\right)^{2}=\sum_{i<j} c_{i} c_{j}\left(y_{i} y_{j}+y_{j} y_{i}\right)+\sum c_{i}^{2} y_{i}^{2}
$$

The latter sum is 0 by definition of special monomial multiplication. If $y_{i} y_{j}$ is the product of more than two indeterminates, then

$$
c_{i} c_{j}\left(y_{i} y_{j}+y_{j} y_{i}\right)=2 c_{i} c_{j} y_{i} y_{j}=0,
$$

since either $y_{i} y_{j}=0$ or $2 c_{i} c_{j}$ is taken mod 2. This completes the proof.

THEOREM 3.3. If $a_{n} \neq \infty$ and $i$ is the least integer for which $a_{i}$ is even (except that if $a_{n}$ is odd, put $\left.i=n\right)$, then $R_{F}\left(a_{1}, \cdots, a_{n}\right.$ ) has order

$$
a_{1}^{n} a_{2}^{n-1} \cdots a_{n}^{1} 2^{2^{n-i+1}-1} 2^{-\langle n-i+1)(n-i+2) / 2} .
$$

Proof. In general there are $\left(\begin{array}{l}n-j \\ k-1\end{array}\right)$ distinct special monomials with $k$ factors such that $j$ is the least subscript appearing among the factors. Such a monomial has order $a_{j}$ if $k \leqq 2$, while if $k>2$ the monomial has order 2 when $a_{j}$ is even and vanishes when $a_{j}$ is odd. Thus the order of $R_{F}$ is given by

$$
\begin{gathered}
\left(a_{1} a_{2} \cdots a_{n}\right)\left(a_{1}^{n-1} a_{2}^{n-2} \cdots a_{n-1}\right)\left[2^{\left(\begin{array}{c}
n-i \\
2
\end{array}\right)+\left(\begin{array}{c}
n-i-1 \\
2
\end{array}\right)+\cdots+\left(\begin{array}{c}
2 \\
2
\end{array}\right)}\right] . \\
\cdot\left[2^{\left(\begin{array}{c}
n-i \\
3
\end{array}\right)+\left(\begin{array}{c}
n-i-1 \\
3
\end{array}\right)+\cdots\left(\begin{array}{c}
3 \\
3
\end{array}\right)}\right] \cdots\left[2^{\left(\begin{array}{c}
n-i \\
n-i
\end{array}\right)}\right] \\
=a_{1}^{n} a_{2}^{n-1} \cdots a_{n}^{1} 2^{2^{n-i-1}-1} 2^{-(n-\imath+1)(n-i+2) / 2},
\end{gathered}
$$

as asserted.

The next theorem elucidates the "free" nature of $R_{F}$.

THEOREM 3.4. If $R$ is a zero-square ring with a standard set of ring generators $x_{1}^{\prime}, \cdots, x_{n}^{\prime}$ of orders $\alpha_{1}^{\prime}, \cdots, a_{n}^{\prime}$, and if $R_{F}\left(a_{1}, \cdots, a_{n}\right)$ is a free zero-square ring with $a_{i}^{\prime} \mid a_{i}$ for $1 \leqq i \leqq n$ (with the convention that every integer and $\infty$ are divisors of $\infty)$, then $R$ is a homomorphic image of $R_{F}\left(a_{1}, \cdots, a_{n}\right)$. 
Proof. Let $x_{1}, \cdots, x_{n}$ be the indeterminates (generators) of $R_{F}$. Let $y_{1}, \cdots, y_{r}$ be the special monomials of $R_{F}$ and $y_{1}^{\prime}, \cdots, y_{r}^{\prime}$ the corresponding monomials of $R$, so that if $y_{i}=x_{i_{1}} \cdots x_{i_{g}}$, then $y_{i}^{\prime}=x_{i_{1}}^{\prime} \cdots x_{i_{g}}^{\prime}$. (Of course for some $i$ we may have $y_{i}^{\prime}=0$.) We then claim that the mapping $\varphi: \sum c_{i} y_{i} \rightarrow \sum c_{i} y_{i}^{\prime}$ is the desired homomorphism.

Since $\alpha_{i}^{\prime} \mid a_{i}$, the ring of integers $\bmod \alpha_{i}^{\prime}$ is a homomorphic image of the ring of integers mod $a_{i}$. It follows from its definition that $\varphi$ preserves sums and products. It remains only to verify that $\phi$ is onto $R$, i.e., that every element of $R$ occurs among $\sum c_{i} y_{i}^{\prime}, 0 \leqq c_{i}<b_{i}$ if $b_{i} \neq \infty,-\infty<c_{i}<\infty$ if $b_{i}=\infty$. This, however, is an immediate consequence of the fact that $R$ is anti-commutative and satisfies $R^{n+1}=0$ and $2 R^{3}=0$, and that the order of an anti-commutative product cannot exceed the g.c.d. of the orders of its factors. This completes the proof.

In general, a subring (or ideal) of $R_{F}\left(a_{1}, \cdots, a_{n}\right)$ need not be free. For instance, if $n>2$ and each $a_{i}$ is even, then $R_{F}^{[(n / 2)+1]}$ is a null ideal with more than one generator.

If $R_{F}=R_{F}\left(a_{1}, \cdots, a_{n}\right)$ is a free zero-square ring such that $i$ is the least integer for which $a_{i}$ is even or $\infty$, and if $n-i \geqq 1$, then it is easily verified that $R_{F}$ has index of nilpotence $n-i+2$. Thus free zero-square rings provide examples of zero-square rings with arbitrarily large index of nilpotence.

4. Nonnull finite zero-square rings. In this section we characterize the additive groups of nonnull finite zero-square rings and as a corollary characterize the orders of such rings. For this purpose we introduce a function $f(G)$ of a finitely generated abelian group $G$.

Definition 4.1. If $G$ is a finitely generated abelian group, define $f(G)=\max \left\{n: R\right.$ is a zero-square $\operatorname{ring}, R^{n} \neq 0, G$ is isomorphic to the additive group $R_{+}$of $R$.

It follows from the local nilpotence of zero-square rings that $f(G)$ is finite. In this section and the next we assume $G$ is finite to avoid looking at a large number of cases. The results can easily be extended to arbitrary finitely generated $G$.

THEOREM 4.2. Let $G$ be a finite abelian group. Then $f(G) \geqq 2$ if and only if either of the following hold:

(i) The dimension of $G$ is greater than two; or

(ii) $G=C_{a_{1}} \oplus C_{a_{2}}$, where $a_{1} \mid a_{2}$ and either $\left(a_{2} / a_{1}, a_{1}\right) \neq 1$ or $a_{1}$ is divisible by a square $>1$. (This condition on $a_{1}$ and $a_{2}$ is equivalent to $a_{1} \mid a_{2}$ and the existence of an integer $b, 0<b<a_{2}$, such that $a_{2} \mid b\left(a_{1}, b\right)$.) 
Proof. We first prove sufficiency of (i) and of (ii). Assume that $G=C_{a_{1}} \oplus C_{a_{2}} \oplus \cdots \oplus C_{a_{n}}$, with $a_{i} \mid a_{i+1}$ and $n \geqq 3$. Let $Z$ be the null ring with additive group $C_{a_{4}} \oplus C_{a_{5}} \oplus \cdots \oplus C_{a_{n}}$. Let $x_{1}, x_{2}$ be generators for the free ring $R_{F}\left(a_{2}, a_{3}\right)$, and let $J$ be the ideal of $R_{F}$ generated by $a_{1} x_{1} x_{2}$. Then it is easily seen that the ring $\left(R_{F} / J\right) \oplus Z$ is a nonnull zero-square ring with additive group isomorphic to $G$. This proves the sufficiency of (i).

The equivalency of the two conditions in (ii) can be verified straightforwardly. To prove the sufficiency of (ii), assume that $G=$ $C_{a_{1}} \oplus C_{a_{2}}$ where $a_{1}$ and $a_{2}$ satisfy the conditions of (ii). In view of Theorem 3.4 we need to prove that if $R_{F}\left(a_{1}, a_{2}\right)$ is generated by $x_{1}, x_{2}$, then the ideal $J$ generated by $x_{1} x_{2}-b x_{2}$ does not contain $x_{1} x_{2}$, where $b$ is defined in (ii). Assume to the contrary that $x_{1} x_{2} \in J$. Then for some $y \in R_{F}$ and some integer $c$,

$$
x_{1} x_{2}=c\left(x_{1} x_{2}-b x_{2}\right)+y\left(x_{1} x_{2}-b x_{2}\right) .
$$

Since $y\left(x_{1} x_{2}-b x_{2}\right)$ contains no term in $x_{2}$, we must have $c b x_{2}=0$. This means $a_{2} \mid b c$. The remaining way an $x_{1} x_{2}$ term can appear is for $y=d x_{1}$. Thus we get

$$
x_{1} x_{2}=(c-b d) x_{1} x_{2} .
$$

We therefore have $\left(a_{1}, c-b d\right)=1$, since the order of $x_{1} x_{2}$ in $R_{F}\left(a_{1}, a_{2}\right)$ is $a_{1}$. This implies $\left(a_{1} b, b c-b^{2} d\right)=b$. We have just proved $a_{2} \mid b c$, and from $a_{2} \mid b\left(a_{1}, b\right)$ we get $a_{2} \mid a_{1} b$ and $a_{2} \mid b^{2}$. Thus $a_{2} \mid\left(a_{1} b, b c-b^{2} d\right)$, or $a_{2} \mid b$, contradicting $0<b<a_{2}$. This proves the sufficiency of (ii).

If $G$ has one generator, then $R$ is clearly null. Hence to prove necessity, we need to show that if $R$ is generated by $x_{1}, x_{2}$ of orders $a_{1}, a_{2}$ with $a_{1} \mid a_{2}$ and $R^{2} \neq 0$, then $a_{1}$ and $a_{2}$ satisfy the conditions in (ii). Let

$$
x_{1} x_{2}=b_{1} x_{1}+b_{2} x_{2}
$$

in $R$. Without loss of generality it may be assumed that $0 \leqq b_{1}<a_{1}$, $0 \leqq b_{2}<a_{2}$.

Assume first that $b_{2}=0$. Then $x_{1} x_{2}=b_{1} x_{1}$, so $0=x_{1} x_{2}^{2}=b_{1} x_{1} x_{2}=$ $b_{1}^{2} x_{1}$; hence $a_{1} \mid b_{1}^{2}$. If $a_{1}$ is divisible by a square $>1$, we have satisfied one of the conditions. Otherwise $b_{1}=0$ since $b_{1}<a_{1}$. In this case $R$ is null, a contradiction.

Now consider the remaining case $x_{1} x_{2}=b_{1} x_{1}+b_{2} x_{2}, b_{2} \neq 0$. Let $c$ be the order of $x_{1} x_{2}$. Then from $0=c x_{1} x_{2}=c b_{1} x_{1}+c b_{2} x_{2}$, we get $0=c b_{1} x_{1}=c b_{2} x_{2}$, so $a_{2} \mid c b_{2}$. Moreover, $0=x_{1}^{2} x_{2}=b_{2} x_{1} x_{2}$ gives $c \mid b_{2}$. Thus $a_{2} \mid b_{2}^{2}$. But $a_{1} x_{1} x_{2}=a_{1} b_{2} x_{2}$ gives $a_{2} \mid a_{1} b_{2}$. Then from $a_{2} \mid b_{2}^{2}$ and $a_{2} \mid a_{1} b_{2}$ we deduce $a_{2} \mid b_{2}\left(a_{1}, b_{2}\right)$. Since $b_{2} \neq 0$, we can take $b=b_{2}$. This completes the proof. 
COROLlaRY 4.3. There exists a nonnull finite zero-square ring of order $r$ if and only if $r$ is divisible by a cube.

COROLLARY 4.4. The smallest nonnull zero-square ring has order 8.

A simple direct proof of Corollary 4.4 is given in [2], (see also Th. 5.7.) It can be shown that there are exactly two nonisomorphic nonnull zero-square rings of order 8 . One of these is $R_{F}(2,2)$.

5. Additive group structure of finite zero-square rings. In this section we extend Theorem 4.2 by considering conditions on $G$ which make $f(G) \geqq n$ for $n>2$. Theorem 5.5 gives some necessary conditions, while Theorem 5.6 provides a converse.

$R$ will denote a finite zero-square ring and $R_{+}$its additive group, while $G$ denotes a finite abelian group and $G_{2}$ its Sylow 2-subgroup. Let $x_{1}, x_{2}, \cdots, x_{n}$ be a fixed standard set of ring generators of $R$. Let $x$ denote the element $x_{1} x_{2} \cdots x_{n}$ and $\overline{x_{i}}$ the element $x_{1} x_{2} \cdots x_{i-1} x_{i+1} \cdots x_{n}$. More generally, if $y=x_{i_{1}} x_{i_{2}} \cdots x_{i_{m}}, i_{1}<i_{2}<\cdots<i_{m}$, then $\bar{y}$ denotes the element $x_{j_{1}} x_{j_{2}} \cdots x_{j_{n-m}}, j_{1}<j_{2}<\cdots<j_{n-m}$, such that the $i$ 's and $j$ 's include all the integers $1,2, \cdots, n$. When $n>2$ note that $y \bar{y}=x$. If $x \neq 0$, we call $m$ the length of $y$, denoted by $|y|$. Note $|y|+|\bar{y}|=n$. If $z \in R$, then $c(z)$ denotes the additive order of $z$.

LEMMA 5.1. Every symmetric matrix of odd order over $G F(2)$ with 0's on the main diagonal is singular.

The proof is a straightforward application of the definition of the determinant and will be omitted.

Lemma 5.2. If a matrix $E$ has the form

$$
E=\left(\begin{array}{cccc}
E_{1} & & & 0 \\
& E_{2} & & \\
& & \ddots & \\
* & & & E_{t}
\end{array}\right),
$$

where the $E_{k}$ are square matrices and some $E_{j}$ is singular, then $E$ is singular.

Proof. This is a special case of the well-known result $\operatorname{det} E=$ $\left(\operatorname{det} E_{1}\right) \cdots\left(\operatorname{det} E_{t}\right)$. 
The next theorem reduces the problem of evaluating $f(G)$ to the case where $G$ is a 2 -group.

ThEOREM 5.3. If $G$ is a finite abelian group and $f(G) \geqq 3$, then $f(G)=f\left(G_{2}\right)$.

Proof. Let $R$ be a finite zero-square ring with $R^{n} \neq 0, n \geqq 3$. By anti-commutativity the elements $R^{\prime}$ of $R$ whose additive order is a power of two form a subring. If $z_{i} \in R, 1 \leqq i \leqq n$, such that $z_{1} z_{2} \cdots z_{n} \neq 0$, where $c\left(z_{i}\right)=a_{i} 2^{b_{i}}, a_{i}$ odd, then $a_{i} z_{i} \in R^{\prime}$ and

$$
\left(a_{1} z_{1}\right)\left(a_{2} z_{2}\right) \cdots\left(a_{n} z_{n}\right) \neq 0
$$

since $2 z_{1} z_{2} \cdots z_{n}=0$. Hence $\left(R^{\prime}\right)^{n} \neq 0$, so $f\left(G_{2}\right) \geqq f(G)$.

Conversely, assume $R^{n} \neq 0$ and $R_{+}$is a 2-group. If $G$ is a finite abelian group with $G_{2} \cong R_{+}$, write $G=G_{2} \oplus H$, and let $S$ be the null ring with $S_{+} \cong H$. Then $(R \oplus S)_{+} \cong G$ and $(R \oplus S)^{n} \neq 0$, so that $f\left(G_{2}\right) \leqq f(G)$. Thus $f(G)=f\left(G_{2}\right)$ and the theorem is proved.

We can now assume in what follows that the additive group $R_{+}$ of $R$ is a 2-group.

LEMMA 5.4. Let $R$ be a finite zero-square ring (with $R_{+}$a 2-group) with $n \geqq 3$ elements $x_{1}, \cdots, x_{n}$ satisfying $x=x_{1} \cdots x_{n} \neq 0$.

(i) There exists a standard set of group generators for $R_{+}$ containing every special monomial $y_{j}$ in the $x_{i}$ of length $3 \leqq\left|y_{j}\right| \leqq n-2$.

(ii) The group generated by those $y_{j}$ satisfying $1 \leqq\left|y_{j}\right| \leqq n-2$ is generated irredundantly by them (though not necessarily standardly).

(iii) If we assume $x_{1}, \cdots, x_{n}$ is a standard set of ring generators for the ring $R^{\prime}$ they generate, then there exists a standard set of group generators for $R_{+}^{\prime}$ containing every special monomial $y_{j}$ in the $x_{i}$ satisfying $\left|y_{j}\right|=1$ or $3 \leqq\left|y_{j}\right| \leqq n-2$.

Proof. (i) If $G$ is a finite abelian $p$-group and $t_{1}, \cdots, t_{s} \in G$, then $t_{1}, \cdots, t_{s}$ extend to a standard set of group generators for $G$ if and only if the following two condition are satisfied:

(1) For any integers $a_{1}, \cdots, a_{s}$,

$$
\sum_{i=1}^{s} a_{i} t_{i}=p z \Rightarrow p \sum_{i=1}^{s} b_{i} t_{i}=p z,
$$

for some integers $b_{1}, \cdots, b_{s}$.

(2) For any integers $a_{1}, \cdots, a_{s}$,

$$
\sum_{i=1}^{s} a_{i} t_{i}=0 \Rightarrow a_{i} t_{i}=0 \text {, all } i \text {. }
$$


To prove (1) in our case, assume

$$
\sum_{3 \leqq\left|y_{i}\right| \leqq n-2} a_{i} y_{i}=2 z
$$

Since $2 y_{i}=0$ when $\left|y_{i}\right| \geqq 3$, we can take $a_{i}=0$ or 1 . Let $y_{j}$ be a special monomial of minimal length satisfying $a_{j}=1$. Then from (3) we get

$$
x=\overline{y_{j}} \sum_{3 \leqq \mid y_{i} \leqq n-2} a_{i} y_{i}=2\left(\overline{y_{j}} z\right)=0,
$$

since $\overline{y_{j}} z \in R^{3}$ when $\left|y_{j}\right| \leqq n-2$. This contradicts $x \neq 0$ and proves (1).

To prove (2), assume

$$
\sum_{3 \leqq\left|y_{i}\right| \leqq n-2} a_{i} y_{i}=0,
$$

where at least one $a_{i} y_{i} \neq 0$. As in (1), let $y_{j}$ be of minimal length such that $a_{j} y_{j} \neq 0$. Multiplying (4) by $\bar{y}_{j}$ gives the contradiction $x=0$, and completes the proof of (i).

(ii) We need to prove that

$$
\sum_{1 \leqq\left|y_{i}\right| \leqq n-2} a_{i} y_{i}=0 \Rightarrow a_{i}=2 b_{i} \text { all } i \text {. }
$$

Letting $y_{j}$ be of minimal length such that $a_{i} \neq 2 b_{i}$ for any integer $b_{i}$, an argument similar to those used in (i) leads to a contradiction.

(iii) We must show (1) and (2) hold, where the $t_{i}$ 's are the $y_{j}$ 's satisfying $\left|y_{j}\right|=1$ or $3 \leqq\left|y_{j}\right| \leqq n-2$, and $p=2$. The proof of (1) is similar to the proof of (5). To prove (2), assume that

$$
\sum_{i=1}^{n} a_{i} x_{i}+\sum_{3 \leqq\left|y_{j}\right| \leqq n-2} b_{j} y_{j}=0 .
$$

By (1), each $b_{j} y_{j}=0$. It follows that each $a_{i} x_{i}=0$ since $x, \cdots, x_{n}$ is a standard set of ring generators. This completes the proof of the lemma.

We can now give necessary conditions for $f(G) \geqq n \geqq 4$.

THEOREM 5.5. Let $G$ be a finite abelian 2-group.

( i ) If $f(G) \geqq n \geqq 4$, then the dimension of $G$ is at least $2^{n}-2[(n+2) / 2]$, i.e., every generating set of $G$ has at least $2^{n}-2[(n+2) / 2]$ elements. (Brackets denote the integer part.)

(ii) If $f(G) \geqq n \geqq 4$, then at least $2^{n}-n(n+1) / 2-2[(n+2) / 2]$ generators in a standard set of generators for $G$ have order 2 .

Proof. (i) Suppose $R$ is a zero-square ring with $R^{n} \neq 0$ and $R_{+} \cong G$, and that $x_{1} x_{2} \cdots x_{n} \neq 0$ in $R(n \geqq 4)$. Let $R^{\prime}$ be the subring 
of $R$ generated by $x_{1}, x_{2}, \cdots, x_{n}$. Since $\operatorname{dim} R_{+}^{\prime} \leqq \operatorname{dim} R_{+}$, it suffices to show $\operatorname{dim} R_{+}^{\prime} \geqq 2^{n}-2[(n+2) / 2]$. By Lemma 5.4 (ii), $\operatorname{dim} R_{+}^{\prime}$ is equal to at least the number of special monomials $y_{i}$ in the indeterminates $x_{1}, \cdots, x_{n}$ satisfying $1 \leqq\left|y_{i}\right| \leqq n-2$. The number of such special monomials is $\left(\begin{array}{l}n \\ 1\end{array}\right)+\left(\begin{array}{c}n \\ 2\end{array}\right)+\cdots+\left(\begin{array}{c}n \\ n-2\end{array}\right)=2^{n}-n-2$. Hence to complete the proof of (i) we need only to prove that when $n$ is odd, we cannot have $\operatorname{dim} R_{+}^{\prime}=2^{n}-n-2$.

Assume that $n$ is odd and $R_{+}^{\prime}$ has $2^{n}-n-2$ generators, $R^{\prime n} \neq 0$. By Lemma 5.4 there is a standard set of group generators for $R_{+}^{\prime}$ containing (1) $x_{1}, \cdots, x_{n},(2)$ all special monomials $y_{j}$ in the $x_{i}$ satisfying $3 \leqq\left|y_{j}\right| \leqq n-2$, and (3) a standard set of generators $y_{1}^{\prime}, \cdots, y_{m}^{\prime}\left(m=\left(\begin{array}{l}n \\ 2\end{array}\right)\right)$ for the group generated by all $y_{j}$ of length 2 . Since this accounts for $2^{n}-n-2$ generators, these in fact generate all of $R_{+}^{\prime}$. In particular the special monomials $\overline{x_{1}}, \cdots, \overline{x_{n}}$ of length $n-1$ can be written as

$$
\bar{x}_{j}=\sum_{1 \leqq\left|y_{i}\right| \leqq n-2} b_{i j} y_{i}, \quad j=1, \cdots, n,
$$

where $b_{i j}$ is an integer. (This representation may not be unique since the $y_{i}$ 's of length 2 need not be standard generators.)

We show that $b_{i j}$ is even. Let $y_{k}$ be a term appearing on the right side of (6) whose coefficient $b_{k_{j}}$ is odd, such that no $y_{i}$ of smaller length has an odd coefficient. Then we get $0=\bar{x}_{j} \bar{y}_{k}=b_{k_{j}} y_{k} \bar{y}_{k}=x$, a contradiction, so every $b_{i j}$ is even. In particular, the terms $b_{i j} y_{i}$ with $\left|y_{i}\right| \geqq 3$ vanish since $2 R^{\prime 3}=0$. If we re-express $\overline{x_{j}}$ as a linear combination of the standard generators given above, then the terms $b_{i j} y_{i}$ with $\left|y_{i}\right|=1$ remain the same. Since $2 \overline{x_{j}}=0$ when $n>3$, we have $b_{i j}=1 / 2 c\left(y_{i}\right)$ or $b_{i j}=0$ whenever $\left|y_{i}\right|=1$. (This is where the argument fails for $n=3$.) Hence we can rewrite (6) as

$$
\bar{x}_{j}=2 \alpha_{j}+\sum_{i=1}^{n} h_{i j}\left(\frac{1}{2} c\left(x_{i}\right)\right) x_{i},
$$

where

$$
2 z_{j}=\sum_{\left|y_{i}\right|=2} b_{i j} y_{i}
$$

and where $h_{i j}=0$ or 1 .

We claim that the matrix $H=\left(h_{i j}\right)$ over $G F(2)$ is nonsingular. If $H$ were singular, then if we regard $1 / 2 c\left(x_{j}\right) x_{j}$ as indeterminates over $G F(2)$, we can eliminate them from (7) and get a relation of the form

$$
\sum_{i=1}^{n} r_{i}\left(\overline{x_{i}}+2 z_{i}\right)=0
$$


where some $r_{j}=1$. But then

$$
x=x_{j} \sum_{i=1}^{n} r_{i}\left(\overline{x_{i}}+2 z_{i}\right)=x_{j} \cdot 0=0,
$$

a contradiction. Hence $H$ is nonsingular.

Therefore we can solve (7) for the $n$ unknowns $1 / 2 c\left(x_{j}\right) x_{j}$ over $G F(2)$ to get

$$
\frac{1}{2} c\left(x_{j}\right) x_{j}=2 \sum_{i=1}^{n} e_{i j} z_{i}+\sum_{i=1}^{n} e_{i j} \overline{x_{i}}, \quad j=1, \cdots, n,
$$

where each $e_{i j}=0$ or 1 . If $E$ denotes the matrix $\left(e_{i j}\right)$ over $G F(2)$, then $E=H^{-1}$, so $E$ is nonsingular.

We will now reach a contradiction by showing that $E$ is singular. We first show $e_{j j}=0$. We have

$$
0=\frac{1}{2} c\left(x_{j}\right) x_{j}^{2}=2 \sum_{i=1}^{n} e_{i j} z_{i} x_{j}+\sum_{i=1}^{n} e_{i j} \overline{x_{i}} x_{j}=e_{j j} x .
$$

Since $x \neq 0, e_{j j}=0$.

Define $s_{1}, s_{2}, \cdots, s_{t}$ by

$$
\begin{aligned}
c\left(x_{1}\right) & =c\left(x_{2}\right)=\cdots=c\left(x_{s_{1}}\right) \\
& <c\left(x_{s_{1}+1}\right)=c\left(x_{s_{1}+2}\right)=\cdots=c\left(x_{s_{2}}\right) \\
& <\cdots<c\left(x_{s_{t-1}+1}\right)=\cdots=c\left(x_{s_{t}}\right),
\end{aligned}
$$

where $s_{t}=n$. Let $E_{k}$ be the square submatrix of $E$ defined by $E_{k}=\left(e_{i j}\right), s_{k-1}+1 \leqq i, j \leqq s_{k}$, for $k=1,2, \cdots, t$. (Here $s_{0}$ is taken to be 0 .) We show that each $E_{k}$ is symmetric. Assume $e_{i j}=1$ for some $s_{k-1}+1 \leqq i, j \leqq s_{k}$. Then from (8) we get $1 / 2 c\left(x_{j}\right) x_{i} x_{j}=x$, so $1 / 2 c\left(x_{j}\right) x_{i} x_{j} \neq 0$. But $1 / 2 c\left(x_{j}\right)=1 / 2 c\left(x_{i}\right)$, as $s_{k-1}+1 \leqq i, j \leqq s_{k}$. Hence $1 / 2 c\left(x_{i}\right) x_{i} x_{j} \neq 0$. From (8) we again get $0 \neq 1 / 2 c\left(x_{i}\right) x_{i} x_{j}=e_{j i} x$, so $e_{j i}=1$. This proves that $E_{k}$ is symmetric. Moreover, $E_{k}$ has 0 's on the main diagonal since each $e_{j j}=0$.

We now show that if for some $k$ we have $i \leqq s_{k}, j>s_{k}$, then $e_{i j}=0$. As in the previous paragraph we have

$$
\frac{1}{2} c\left(x_{j}\right) x_{2} x_{j}=e_{i j} x
$$

Since $i \leqq s_{k}, j>s_{k}$ we have $c\left(x_{i}\right)<c\left(x_{j}\right)$. Therefore $1 / 2 c\left(x_{i}\right) x_{i} x_{j} \neq 0$. But from (4), $1 / 2 c\left(x_{i}\right) x_{i} x_{j}=e_{j i} x$, so $c\left(x_{i}\right) x_{i} x_{j}=2 e_{j i} x=0$ (since $2 x=0$ ). But $c\left(x_{i}\right)<c\left(x_{j}\right)$ implies $c\left(x_{i}\right) \leqq 1 / 2 c\left(x_{j}\right)$, so $1 / 2 c\left(x_{j}\right) x_{i} x_{j}=0$. Comparing with (9) shows $e_{i j}=0$, as asserted.

This shows that $E$ has the form given in Lemma 5.2. Since the 
sum of the orders of the $E_{j}$ must be the order of $E$, some $E_{k}$ has odd order. Then by Lemma $5.1 E_{k}$ is singular, so by Lemma $5.2 E$ is singular, a contradiction. This completes the proof of (i).

(ii) Using the notation of part (i), it follows from $2 R^{\prime 3}=0$ that every special monomial $y_{i}$ satisfying $3 \leqq\left|y_{i}\right| \leqq n-2$ has order 2 . There are $\left(\begin{array}{l}n \\ 3\end{array}\right)+\left(\begin{array}{l}n \\ 4\end{array}\right)+\cdots+\left(\begin{array}{c}n \\ n-2\end{array}\right)=2^{n}-n(n+1) / 2-(n+2)$ such $y_{i}$, and by Lemma 5.4 they extend to a standard set of group generators for $R_{+}$. Moreover, we have just shown that when $n$ is odd, there is at least one $y_{j}$ with $\left|y_{j}\right|=n-1$ which cannot be expressed in the form $y_{j}=\sum_{1 \leqq\left|y_{i}\right| \leqq n-2} s_{i} y_{i}$. Exactly as in the proof of Lemma 5.4 it follows that the set of all $y_{i}$ satisfying $3 \leqq\left|y_{i}\right| \leqq n-2$, along with $y_{j}$, extend to a standard set of group generators for $R_{+}$. Thus we have found $2^{n}-n(n+1) / 2-2[(n+2) / 2]$ generators of order 2, proving (ii) and completing the proof of the theorem.

The following theorem shows that the results of the previous theorem are best possible.

THEOREM 5.6. Let $n \geqq 4$ be an integer.

(i ) Given any integer $N \geqq 2^{n}-2[(n+2) / 2]$, there exists a finite abelian 2-group $G$ of dimension $N$, such that $f(G)=n$.

(ii) Given any integer $M \geqq 2^{n}-n(n+1) / 2-2[(n+2) / 2]$, there exists a finite abelian 2-group $G$ with precisely $M$ generators of order 2 (in a standard set of generators), such that $f(G)=n$.

Proof. Clearly to prove both (i) and (ii) it suffices to construct a finite zero-square ring $R$ with $R^{n} \neq 0(n \geqq 4)$, such that $R_{+}$has precisely $N=2^{n}-2[(n+2) / 2]$ standard group generators, with precisely $M=2^{n}-n(n+1) / 2-2[(n+2) / 2]$, of these generators of order 2 . Let $m=[n / 2]$ and let $R_{F}\left(a_{1}=8, a_{2}=8, \cdots, a_{n}=8\right)$ be a free ring with generators $x_{1}, \cdots, x_{n}$ (as defined in $\S 3$ ). If $n$ is even let $J$ be the ideal generated by $\left\{\bar{x}_{1}-4 x_{2}, \bar{x}_{2}-4 x_{1}, \bar{x}_{3}-4 x_{4}, \bar{x}_{4}-4 x_{3}, \cdots, \bar{x}_{n-1}-4 x_{n}, \bar{x}_{n}-4 x_{n-1}\right\}$, while if $n$ is odd let $J$ be generated by $\left\{\bar{x}_{1}-4 x_{2}, \bar{x}_{2}-4 x_{1}, \cdots\right.$, $\left.\bar{x}_{n-2}-4 x_{n-1}, \bar{x}_{n-1}-4 x_{n-2}\right\}$. Let $R=R_{F} / J$. Then $R$ is generated by the images of all $y_{i}$ satisfying $1 \leqq\left|y_{i}\right| \leqq n-2$ when $n$ is even; with the additional generator $\bar{x}_{n}$ when $n$ is odd. This gives a total of $2^{n}-2[(n+2) / 2]$ generators, as desired. Moreover, when $n$ is even, a standard set of group generators for $R_{+}$has $n+1$ elements of order $8,2 m^{2}-m-1$ elements of order 4 , and exactly $M$ elements of order 2. When $n$ is odd, there are $n+1$ elements of order $8,2 m^{2}+m-1$ elements of order 4 , and exactly $M$ elements of order 2 . Hence it remains to prove that the image of $x$ in $R_{F} / J$ is not 0 , i.e., that $x \notin J$. We treat only the case when $n$ is even; the case $n$ odd is 
almost exactly the same.

Assume $x \in J$. Then

$$
\begin{aligned}
x= & z_{1}\left(\bar{x}_{1}-4 x_{2}\right)+z_{2}\left(\bar{x}_{2}-4 x_{1}\right)+\cdots+z_{n}\left(\bar{x}_{n}-4 x_{n-1}\right) \\
& +b_{1}\left(\bar{x}_{1}-4 x_{2}\right)+b_{2}\left(\bar{x}_{2}-4 x_{1}\right)+\cdots+b_{n}\left(\bar{x}_{n}-4 x_{n-1}\right),
\end{aligned}
$$

where $z_{i} \in R_{F}, b_{i}=0$ or 1 . Hence we need at least one $z_{j}=x_{j}$, say $z_{1}=x_{1}$. We then also get the term $-4 x_{1} x_{2}$, which can only be cancelled by $z_{2}=x_{2}$, giving another $-4 x_{1} x_{2}$. But this also gives another $x$, and $x+x=0$. Hence $x \in J$, and the theorem is proved.

REMARK. The proofs of Theorems 5.5 and 5.6 are not valid for $n=3$, basically because from $\left|y_{i}\right|=n-1$ we cannot deduce $2 y_{i}=0$. If Theorem 5.5 (i) were false for $n=3$, then there would be a 2-group $G$ with three generators such that $f(G)=3$. Although this seems highly unlikely, the question remains open. Clearly $G$ cannot have less than three generators. Note that Theorem 5.5 (ii) is trivially satisfied for $n=3$. Finally, Theorem 5.6 is easy to verify for $n=3$ (though in part (ii) we of course must have $M \geqq 0$ ).

It is considerably simpler to get results on the order of zero-square rings satisfying $R^{n} \neq 0$.

THeOREm 5.7. Assume $n>2$. Then there exists a zero-square ring of order $r$ satisfying $R^{n} \neq 0$ if and only if $2^{2^{n-1}} \mid r$.

Proof. Assume $R^{n} \neq 0$. We know from the proof of Theorem 5.3 that there are elements $x_{1}, \cdots, x_{n}$ in the Sylow 2-subgroup $R_{2}$ of $R_{+}$such that $x_{1} \cdots x_{n} \neq 0$. Let $y_{1}, y_{2}, \cdots, y_{2^{n}-1}$ be the special monomials in the $x_{i}$. Claim that the $2^{2^{n}-1}$ elements of the form $\sum_{i=1}^{2^{n}-1} b_{i} y_{i}, b_{i}=0$ or 1 , are all distinct, otherwise we would have a relation of the form

$$
\sum b_{i} y_{i}=0,
$$

with at least one $b_{i}=1$. Let $y_{j}$ be a special monomial of shortest length such that $b_{j}=1$. Then multiplying (6) by $\bar{y}_{j}$ gives $x=0$, a contradiction. Hence $R_{2}$ has order at least $2^{2^{n}-1}$, so that $2^{2^{n}-1}|| R_{2} \mid$. Hence $2^{2^{n}-1} \mid r$.

Conversely, if $2^{2^{n-1}} \cdot s=r$, then we take $R_{+}$to be $C_{2}^{2^{n}-1} \oplus C_{s}$. If we impose the free ring $R_{F}(2,2, \cdots, 2)$ on $C_{2}^{2^{n}-1}$ and the null ring on $C_{s}$, then $R^{n} \neq 0$.

Finally we have the result of [3].

COROLLARY 5.8. The smallest zero-square ring $R$ satisfying $R^{n} \neq 0$, $n>1$, has order $2^{2^{n-1}}$. 


\section{REFERENCES}

1. A. Abian and W. A. McWorter, On the index of nilpotency of some nil algebras, Boll. Un. Mat. Ital. (3) 18 (1963), 252-255.

2. L. Carlitz, Solution to E 1665, Amer. Math. Monthly 72 (1965), 80.

3. G. A. Heuer, Two undergraduate projects, Amer. Math. Monthly 72 (1965), 945.

Received September 9, 1968. This paper was written for the 1965 Bell prize at the California Institute of Technology, under the guidance of Professor Richard A. Dean.

CALifornia Institute of TeChNology

HARVARD UNIVERSITY 


\section{PACIFIC JOURNAL OF MATHEMATICS}

\section{EDITORS}

H. ROYDEN

Stanford University

Stanford, California

\section{RichaRd PIERCE}

University of Washington Seattle, Washington 98105
J. DugundJI

Department of Mathematics

University of Southern California

Los Angeles, California 90007

BASIL GORDON

University of California

Los Angeles, California 90024

\section{ASSOCIATE EDITORS}
E. F. BECKENBACH
B. H. NeUMANN
F. WOLF
K. YOSHIDA

\section{SUPPORTING INSTITUTIONS}

UNIVERSITY OF BRITISH COLUMBIA CALIFORNIA INSTITUTE OF TECHNOLOGY

UNIVERSITY OF CALIFORNIA

MONTANA STATE UNIVERSITY

UNIVERSITY OF NEVADA

NEW MEXICO STATE UNIVERSITY

OREGON STATE UNIVERSITY

UNIVERSITY OF OREGON

OSAKA UNIVERSITY

UNIVERSITY OF SOUTHERN CALIFORNIA

\author{
STANFORD UNIVERSITY \\ UNIVERSITY OF TOKYO \\ UNIVERSITY OF UTAH \\ WASHINGTON STATE UNIVERSITY \\ UNIVERSITY OF WASHINGTON \\ AMERICAN MATHEMATICAL SOCIETY \\ CHEVRON RESEARCH CORPORATION \\ TRW SYSTEMS \\ NAVAL WEAPONS CENTER
}

The Supporting Institutions listed above contribute to the cost of publication of this Journal, but they are not owners or publishers and have no responsibility for its content or policies.

Mathematical papers intended for publication in the Pacific Journal of Mathematics should be in typed form or offset-reproduced, double spaced with large margins. Underline Greek letters in red, German in green, and script in blue. The first paragraph or two must be capable of being used separately as a synopsis of the entire paper. It should not contain references to the bibliography. Manuscripts, in duplicate if possible, may be sent to any one of the four editors. Please classify according to the scheme of Math. Rev. 36, 1539-1546. All other communications to the editors should be addressed to the managing editor, Richard Arens, University of California, Los Angeles, California, 90024.

50 reprints are provided free for each article; additional copies may be obtained at cost in multiples of 50 .

The Pacific Journal of Mathematics is published monthly. Effective with Volume 16 the price per volume (3 numbers) is $\$ 8.00$; single issues, $\$ 3.00$. Special price for current issues to individual faculty members of supporting institutions and to individual members of the American Mathematical Society: $\$ 4.00$ per volume; single issues $\$ 1.50$. Back numbers are available.

Subscriptions, orders for back numbers, and changes of address should be sent to Pacific Journal of Mathematics, 103 Highland Boulevard, Berkeley, California, 94708.

PUBLISHED BY PACIFIC JOURNAL OF MATHEMATICS, A NON-PROFIT CORPORATION

Printed at Kokusai Bunken Insatsusha (International Academic Printing Co., Ltd.), 7-17. Fujimi 2-chome, Chiyoda-ku, Tokyo, Japan. 


\section{Pacific Journal of Mathematics}

\section{Vol. 30, No. $3 \quad$ November, 1969}

Willard Ellis Baxter, Topological rings with property $(Y) \ldots \ldots \ldots \ldots . \ldots 5$

Sterling K. Berberian, Note on some spectral inequalities of $C . R$.

Putnam ..................................... 573

David Theodore Brown, Galois theory for Banach algebras . ........... 577

Dennis K. Burke and R. A. Stoltenberg, A note on p-spaces and Moore spaces ........................................ 601

Rafael Van Severen Chacon and Stephen Allan McGrath, Estimates of positive contractions....................................... 609

Rene Felix Dennemeyer, Conjugate surfaces for multiple integral problems in the calculus of variations ........................... 621

Edwin O. Elliott, Measures on countable product spaces.............. 639

John Moss Grover, Covering groups of groups of Lie type .............. 645

Charles Lemuel Hagopian, Concerning semi-local-connectedness and cutting in nonlocally connected continua .................. 657

Velmer B. Headley, A monotonicity principle for eigenvalues ........... 663

John Joseph Hutchinson, Intrinsic extensions of rings . . . . . . . . . . . . . 669

Harold H. Johnson, Determination of hyperbolicity by partial

prolongations .................................. 679

Tilla Weinstein, Holomorphic quadratic differentials on surfaces in $E^{3} \ldots 697$

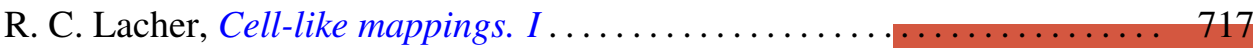

Roger McCann, A classification of centers

Curtis L. Outlaw, Mean value iteration of nonexpansive mappings in a

Banach space...

Allan C. Peterson, Distribution of zeros of solutions of a fourth order

differential equation.

Bhalchandra B. Phadke, Polyhedron inequality and strict convexity .. 765 Jack Wyndall Rogers Jr., On universal tree-like continua .

Edgar Andrews Rutter, Two characterizations of quasi-Frobenius rings

G. Sankaranarayanan and C. Suyambulingom, Some renewal theorems concerning a sequence of correlated random variables...

Joel E. Schneider, A note on the theory of primes........ . .

Richard Peter Stanley, Zero square rings .................

Edward D. Tymchatyn, The 2-cell as a partially ordered space

Craig A. Wood, On general Z.P.I.-rings................ 